DOI: https://doi.org/10.46296/yc.v4i7.0053

\title{
ESTRATEGIAS RESILIENTES Y DROGODEPENDENCIA EN PACIENTES DEL CENTRO DE REHABILITACIÓN NUEVA ESPERANZA DEL CANTÓN BOLÍVAR - MANABÍ.
}

\section{RESILIENT STRATEGIES AND DRUG DEPENDENCE IN PATIENTS OF THE NUEVA ESPERANZA REHABILITATION CENTER OF THE BOLÍVAR CANTON - MANABÍ.}

\author{
Ponce-Zambrano José Arturo ${ }^{1 *}$; Tarazona-Meza Katherine ${ }^{2}$ \\ 1,2 Universidad Técnica de Manabí, UTM. Portoviejo, Ecuador. \\ *Correo: joar.poza29@gmail.com
}

\begin{abstract}
Resumen
El presente estudio se realizó con el objetivo de identificar las estrategias resilientes que desarrollan los pacientes drogodependientes derivados del programa de rehabilitación del centro de rehabilitación Nueva Esperanza del cantón Bolívar - Manabí. Se utilizó un enfoque mixto (cuanticualitativo) de tipo descriptivo y con un método de análisis y síntesis. Donde se realizó un análisis documental de los programas, entrevistas a profundidad con el fin de identificar técnicas de intervención resilientes, posteriormente se realizó la observación no participante con el fin de identificar las actividades que fomentan la resiliencia en los pacientes. También se aplicó la prueba psicométrica SV- RES. Para determinar el nivel y constatar el desarrollo de las capacidades resilientes. La investigación concluye que existen estrategias resilientes al ejecutar distintas terapias como las terapias grupales, individuales, espirituales y ocupacionales, así como técnicas de intervención psicológica que promueven el desarrollo de la resiliencia, sin embargo, en los resultados obtenidos no se observa un aumento de la resiliencia por la falta de personal multidisciplinario, técnicas de intervención y poca la frecuencia en las que se realiza.
\end{abstract}

Palabras Clave: Resiliencia, estrategias, drogodependencia, programa, rehabilitación.

\begin{abstract}
The present study was carried out with the objective of identifying the resilient strategies developed by drug-dependent patients of the rehabilitation program of the Nueva Esperanza rehabilitation center in Bolívar - Manabí canton. A mixed approach (quantitative-qualitative) of a descriptive type and a method of analysis and synthesis was used. Where a documentary analysis of the programs was carried out, in-depth interviews in order to identify resilient intervention techniques, then non-participant observation was carried out in order to identify the activities that promote resilience in patients. The SV-RES psychometric test was also applied. To determine the level and verify the development of resilient capacities. The research concludes that there are resilient strategies when executing different therapies such as group, individual, spiritual and occupational therapies as well as psychological intervention techniques that promote the development of resilience, however, the results obtained do not show an increase in resilience due to the lack of multidisciplinary personnel, intervention techniques and the infrequency in which it is carried out.
\end{abstract}

Keywords: Resilience, strategies, drug dependence, program, rehabilitation.

Información del manuscrito:

Fecha de recepción: 12 de mayo de 2020.

Fecha de aceptación: 09 de julio de 2020.

Fecha de publicación: 10 de julio de 2020. 


\section{Introducción}

El fenómeno mundial de las drogas representa un problema cada vez más complejo, dinámico y de múltiples causas que afecta a la salud, la convivencia social y la seguridad ciudadana, porque desintegra la sociedad y lo más importante desintegra su núcleo familiar. Esta problemática mundial hace que el país se vea en la necesidad de adquirir un enfoque integral, multidisciplinario y rápido que tenga consigo validez científica y que se fomente de forma efectiva en la sociedad. (Secretaria Técnica de Prevención Integral de Drogas, 2017).

Del mismo modo existen ciertos centros de rehabilitación para personas adictas al consumo de drogas, donde se han evidenciado algunas falencias profesionales en la intervención para la rehabilitación de estos pacientes. Se ha identificado cierto índice de instituciones que no cuentan con la infraestructura adecuada, con permisos ni pautas de intervención establecidas por el Ministerio de Salud, que exige establecer un programa integral de intervención para lograr una rehabilitación más eficaz.
Desde el planteamiento de los estudios en drogodependencia se ha analizado los factores protectores como características que ayudan tanto a la persona drogodependientes llevar una mejor rehabilitación, como desde el punto de vista de la prevención de recaídas. Por eso, es relevante mostrar la perspectiva de la resiliencia ya que es uno de los principales enfoques que trabaja desde la protección y no desde el riesgo. (Cabrera, 2019).

Ariza, Rubio, \& Russi, (2015) mencionan que para los drogodependientes esta capacidad de resiliencia no ha sido desarrollada en su totalidad puesto que las adversidades a las que se han sometido tales como: acoso y abuso sexual, pérdida de seres queridos, pobreza extrema, indigencia, violencia y negligencia familiar se han venido evidenciando desde la niñez para algunos y han permanecido en la adolescencia y adultez generando vacíos afectivos familiares y de pareja, duelos no elaborados, prostitución y delincuencia común generada por falta de recursos económicos o influencia de pares negativos que les 
han impedido dar un valor o un sentido a sus vidas lo cual generó el inicio y el mantenimiento del consumo como medio facilitador para evadir la realidad que estaban enfrentando.

Es importante que los programas de rehabilitación de estos centros desarrollen actividades que fomenten la resiliencia en sus pacientes, un ejemplo de ello es el centro de rehabilitación Nueva Esperanza que cuenta con un programa de intervención integral con un personal interdisciplinario en diferentes áreas como la psicología, terapistas espirituales, psiquiatría y terapistas vivenciales, donde se busca una recuperación eficaz y eficiente en los pacientes que han consumido alcohol y otras drogas.

Para comprender esta idea, se precisa profundizar en diferentes términos como la resiliencia donde Grotberg (1995 citado en Villalba, 2004, p.285) lo define como la capacidad universal que permite a las personas, familias, grupos 0 comunidades prevenir, minimizar 0 sobreponerse a los efectos dañinos de la adversidad. La resiliencia está formada por distintos factores protectores que trabajan protegiendo al sujeto de las adversidades encontradas en su entorno.

Estos factores se agrupan en distintos grupos de factores personales como, las relaciones sociales, el humor positivo y el ritmo biológico estable, también se agrupan factores cognitivos como afectivos como la empatía, autonomía en las acciones tomadas y resolución de problemas, existen también factores como las emociones positivas que pueden disminuir y prevenir los efectos de las adversidades. (Oros, 2009 citado por García\& Domínguez, 2013).

También es importante considerar que la rehabilitación se refiere al desarrollo de tratamiento que comienza después de que se completa la desintoxicación; este procedimiento puede realizarse en un hospital o ambulatorio. Por lo general, incluye terapias individuales, grupales y familiares, así como educación psicológica sobre drogas y alcohol, e intenta guiar a los pacientes y sus familias a establecer un entorno social que apoye la abstinencia. Las técnicas de tratamiento están diseñadas para 
ayudar a los pacientes a reducir o suprimir los comportamientos desadaptativos y aprender a prevenir los comportamientos de recaída (NCADI, 2000; Citado en Cano, Nanni, \& Pantoja, 2016).

La mayoría de los programas de rehabilitación utilizan un programa activo de educación psicológica, haciendo hincapié en el uso continuo y a gran escala de drogas en los efectos negativos biológicos, psicológicos y sociales. Además, muchos procedimientos utilizan métodos para enseñar a los pacientes los factores que causan la recaída. No debe subestimarse la importancia de la educación psicológica de los pacientes por parte de psiquiatras y todo el equipo de salud mental, especialmente con respecto a las consecuencias médicas del consumo excesivo de alcohol y drogas (NTIES, 1997; Citado en Cano, Nanni, \& Pantoja, 2016).

Existe una gran relación entre la resiliencia y la drogodependencia, por ejemplo, al momento de estar en la etapa de rehabilitación, los adictos se han mostrado resilientes ante su tratamiento sin importar el trauma que hayan vivido (Pimentel, Telumbre, Ruiz, Higuera, 2015).

Por esta razón la resiliencia es de gran importancia en la vida de las personas con adicciones ya que produce efectos positivos por medio de la regulación saludable de las cogniciones, emociones y acciones que son útiles para crear invulnerabilidad psicológica, por eso es conveniente conocer la resiliencia, que al formar parte de la psicología positiva, es un factor que promueve todas estas características para que el individuo pueda mantenerse estable y vivir en sociedad $\sin$ consecuencias psicológicas o físicas negativas que genera el consumo de sustancias. (Jiménez y Matute, 2018).0

La falta de resiliencia puede generar mayor vulnerabilidad en los pacientes que presentan dependencias a sustancias psicoactivas, por esta razón es importante desarrollar esta capacidad en los pacientes drogodependientes a través de estrategias que fomenten la resiliencia y así mejorar la recuperación y prevenir recaídas, por lo tanto el objetivo de esta investigación es identificar las 
estrategias resilientes que

desarrollan los pacientes drogodependientes derivados del programa de rehabilitación del centro de rehabilitación Nueva Esperanza del cantón Bolívar-Manabí Este aporte investigativo contribuye a la sociedad para mejorar el tratamiento y la prevención de las recaídas en los pacientes drogodependientes.

\section{Materiales y métodos}

La investigación se realizó en el centro de rehabilitación Nueva Esperanza del cantón Bolívar Manabí, durante los tres primeros meses del año 2020, teniendo en cuenta una población y muestra de 15 pacientes que participan en el programa de rehabilitación y 4 terapeutas que laboran en esta institución.

El proceso de investigación se llevó a cabo mediante un enfoque mixto (cuanti-cualitativo), de tipo descriptivo y se utilizó el método de análisis síntesis. Se utilizó como técnicas el análisis documental de los programas, mediante fichas, para identificar las actividades realizadas durante todo el proceso de rehabilitación y que fomentan la resiliencia en los pacientes. También se realizaron entrevistas a profundidad a los terapeutas del centro, donde se establecieron diferentes situaciones específicas que existen durante el proceso de rehabilitación y que permitieron identificar las técnicas de intervención que contribuyen a la formación de resiliencia, posteriormente se realizó la observación no participante, con un registro de los criterios de evaluación de estrategias resilientes en las reuniones terapéuticas. En cuanto al enfoque cuantitativo se aplicó la prueba psicométrica SV- RES (Saavedra \& Villalta, 2008) para determinar el nivel y el desarrollo de las capacidades resilientes, donde se buscó diferenciar los resultados de los pacientes recién participantes del programa y los que cuentan con mayor tiempo dentro del mismo y así constatarlos con lo propuesto en el programa de rehabilitación.

La investigación fue realizada con distintos materiales tecnológicos como: Laptop, calculadora, computadora, el programa de Word, el programa Excel para la tabulación de resultados. 


\section{Resultados}

En el análisis documental del programa que desarrolla el centro de rehabilitación, se buscó sintetizar y esquematizar las actividades que más se destacan y se convierten en estrategias resilientes en los pacientes, dentro de ellas se encuentra el trabajo bajo el modelo transteorico de Proshaska y Diclemente, terapias individuales, terapias grupales, ocupacionales y espirituales, también se realizan terapias cognitivo conductuales, psicoeducación, el entrenamiento de habilidades sociales y técnicas asertivas, técnicas de autocontrol y técnicas de relajación. Mediante las entrevistas a profundidad a los terapeutas y personal que labora en el centro de rehabilitación se pudieron encontrar algunas técnicas de intervención que pueden contribuir a la formación de resiliencia como los proyectos de vida, psicodrama, risoterapia, también se pone en práctica la realización competencias deportivas y se pudo constatar que algunas de las intervenciones descritas en el programa de rehabilitación no se realizan con frecuencia como intervenciones psicológicas y orientaciones familiares. Por otro lado se descubrió la falta de herramientas que ayudan a fomentar la resiliencia en casos específicos como indisciplina, discusiones o altercados donde existe cierta carencia en las intervenciones descubriendo la falta de habilidades como la comunicación asertiva o la empatía, En la observación no participante que se realizó en tres terapias grupales, se pudo constatar la aplicación de distintas técnicas o formas de intervenir que se asocian a la resiliencia, donde se promueve la iniciativa, la creatividad, la introspección y la participación. También se identificó un lenguaje coloquial y falta de asertividad $y$ empatía durante la interacción entre pacientes y terapeutas.

Así también se realizó la aplicación del test psicométrico SV-RES para medir los niveles de resiliencia con el fin de determinar el nivel y el desarrollo de las capacidades resilientes a 15 pacientes del centro de rehabilitación que participaron del programa de rehabilitación como se evidencia en la tabla 1. 
Tabla 1. Resultados de escala SV-RES de Saavedra y Villalta.

\begin{tabular}{lcccc}
\hline $\begin{array}{l}\text { Nivel de } \\
\text { resiliencia }\end{array}$ & $\begin{array}{l}-3 \\
\text { meses }\end{array}$ & $\begin{array}{c}+3 \\
\text { meses }\end{array}$ & $\%$ \\
\hline Alto & 1 & $20 \%$ & 1 & $10 \%$ \\
Medio & 3 & $60 \%$ & 6 & $60 \%$ \\
Bajo & 1 & $20 \%$ & 3 & $30 \%$ \\
Total & 5 & $100 \%$ & 10 & $100 \%$ \\
\hline
\end{tabular}

El programa de rehabilitación tiene una duración de seis meses, en el que se diferenciaron los niveles de resiliencia de los pacientes que recién inician el proceso de rehabilitación con menos de tres meses y los pacientes con más de tres meses en el proceso, determinando que del total de los pacientes con menos de tres meses el $20 \%$ presenta un nivel alto de resiliencia, el $60 \%$ presenta un nivel medio y al $20 \%$ se le atribuye un nivel bajo de resiliencia.

También se observaron los resultados de los pacientes con más de tres meses en el proceso de rehabilitación encontrando un 10\% de los pacientes con un nivel alto en resiliencia, el $60 \%$ con un nivel medio en resiliencia y el $30 \%$ de los pacientes con un nivel bajo en resiliencia.
Por otro lado, se pueden observar los porcentajes de los 12 factores de resiliencia propuestos en el test SVRES, que están incorporados a los aportes de Grotberg (1995) en Munist, et. al. (1998) Citado por Callisaya (2017) En el sentido de abordar los diversos niveles que contribuyen a la formación de la resiliencia como el "Yo tengo" que corresponde al entorno social, el "Yo soy" y "Yo estoy" que se refiere a la fuerza psicológica interna del sujeto y el "Yo puedo" que correspondiente a las habilidades en las relaciones con los otros.

Tabla 2. Factores de la Resiliencia.

\begin{tabular}{cccccc}
\hline $\begin{array}{c}\text { Yo } \\
\text { soy, }\end{array}$ & Identidad & Autonomía & Satisfacción & Pragmatismo & Total \\
yo & $27 \%$ & $25 \%$ & $22 \%$ & $26 \%$ & $100 \%$ \\
estoy. & & & & & \\
\hline Yo & Vínculos & Redes & Modelo & Metas & Total \\
tengo & $24 \%$ & $23 \%$ & $26 \%$ & $26 \%$ & $100 \%$ \\
\hline Yo & Afectividad & Autoeficacia & Aprendizaje & Generatividad & Total \\
puedo & $24 \%$ & $24 \%$ & $26 \%$ & $26 \%$ & $100 \%$ \\
\hline
\end{tabular}

Dentro del nivel "yo soy, yo estoy", se observa en la tabla 2 que el factor de identidad que presenta el porcentaje más alto en los pacientes del centro de rehabilitación con un $27 \%$, que habla sobre los juicios generales que el sujeto define sobre sus valores culturales de un modo estable, le sigue el pragmatismo con un $26 \%$, que refiere a la forma que el sujeto 
interpreta las acciones que realiza. La autonomía con un 25\% que hace referencia a los juicios del vínculo del sujeto consigo mismo y su aporte con el entorno social y la satisfacción con un $22 \%$ que representa a las formas de interpretar las situaciones adversas.

En el nivel "Yo tengo" se distingue un porcentaje de $24 \%$ en vínculos que representa a los juicios del individuo sobre las redes sociales de su historia personal. También se destaca un $23 \%$ en redes que hace referencia a los juicios del individuo con respecto al vínculo afectivo que tiene con el entorno. Se menciona también un $26 \%$ en modelo que son los juicios del sujeto sobre la convicción de las redes sociales cercanas y su apoyo para la superación de situaciones adversas y se observa también un $26 \%$ en metas que son los juicios del valor contextual de las metas y redes sociales sobre la situación problema.

En el nivel "Yo puedo" se observa un $24 \%$ en afectividad que refiere al juicio de las posibilidades sobre sí mismo y su vínculo con los demás. También se evidencia el $24 \%$ en autoeficacia que refiere a las posibilidades de éxito que el sujeto reconoce de sí mismo ante los problemas, también se obtuvo el $26 \%$ en aprendizaje que se refiere al valor del sujeto sobre las situaciones adversas y un posible aprendizaje y un $26 \%$ en generatividad que representa a la posibilidad que el sujeto identifica al pedir ayuda a otras personas en situaciones problemáticas.

\section{Discusión}

Con los resultados obtenidos podemos determinar que existen distintas técnicas o actividades que pueden convertirse en estrategias para desarrollar resiliencia en los pacientes del centro de rehabilitación como el modelo transteórico de Prochaska y Di clemente donde (Prochaska et al., 2015) toma a la autoeficacia como una variable indispensable en su modelo considerándola como una oposición a la tentación y (Saavedra \& Villalta, 2008) la adjunta como factor de resiliencia definiéndola como la capacidad de poner límites, controlar los impulsos, responsabilizarse por los actos, manejar el estrés y terminar lo propuesto ante un problema. Por otra parte se destacan las terapias grupales mencionando a 
(Becoña, 2002; p.2 Citado por Manchini, 2018, p. 36) que demuestra que el propósito de la terapia grupal es el mismo que el de la terapia individual donde cada grupo tiene su propia motivación de desarrollo permitiendo a los pacientes compararse con los demás, obtener apoyo, aprender estrategias de afrontamiento, Autonomía y dominar habilidades sociales, que son variables que se asocian a los factores de las dimensiones de la resiliencia propuestos por Saavedra (2008). También permite ver si el paciente es capaz de seguir normas, reglas, solucionar posibles conflictos, integrarse socialmente y trabajar en equipo lo que conlleva actuar de forma resiliente ante situaciones de presión (Apolo, Rivas, \& Cucalón, 2017).

Otra actividad que fomenta la resiliencia es el psicodrama a través de la creatividad, donde existen técnicas que plantean una predicción del futuro para que las personas se den cuenta de que pueden superar esta adversidad. Por tanto, la técnica cultiva las características de las personas resilientes. Así también, las personas generalmente sienten que no pueden continuar su vida después de experimentar una situación traumática o volver a ser diferentes nuevamente, pero debido a las técnicas del psicodrama, las personas crean diferentes motivaciones y formas, además de percibir la realidad (Lizano, 2017, p. 68). También se destaca la risa y el humor que son un estilo de afrontamiento instintivo que ayuda a enfrentar las desilusiones y desafíos de la vida, a través de ellos se cree que las personas pueden reemplazar las emociones negativas por emociones positivas y ganar optimismo y esperanza de vida, así mismo aumenta la capacidad para lidiar con estados negativos, estresados y amenazadores (Villán, Gaona, \& Carrero, 2018, p. 39).

Otra forma de evidenciar el desarrollo de la resiliencia es la terapia ocupacionales que trata de restablecer, modificar o crear nuevos sentidos y significados a través del uso de la ocupación en los pacientes con problemas de consumo, de modo que a través de la ocupación, podamos determinar el interés del sujeto por su vida, nivel de satisfacción, su entorno laboral, 
actividades que valoraba más 0 dedicaba más tiempo a las actividades, y el grado de identidad del sujeto que posee con los roles y expectativas de la sociedad, entre otras (Farias, Guerra, Cifuentes, \& Rozas, 2010, p.47).

También se puede reconocer a la terapia espiritual como estrategia para desarrollar resiliencia, ya que para Puig y Rubio (2013) citado por Cruz (2016) la espiritualidad es un pilar de la resiliencia explicando que es la capacidad de las personas para darle sentido a su vida proporcionándole razones para trascender, la espiritualidad se desarrolla durante la niñez y encuentra sentido en la adolescencia y adultez. Esta cualidad es indispensable en la recuperación de adictos, porque una vez establecida se convierte en recurso, favoreciendo el bienestar emocional y propiciando la salida reforzada de ese contacto directo con el sufrimiento, haciendo a las personas más resilientes (Benito, Barbero et al., 2014; Frankl, 1996 citado por Redondo et al., 2017).

Otro aporte importante para destacar son los estudios de Campos \& Odar (2018) afirmando que el programa cognitivo conductual tiene un efecto grande en mejorar la resiliencia de internos en un centro de rehabilitación. En relación con esta posición la terapia cognitivo conductual suele incluir una serie de estrategias destinadas a mejorar el autocontrol. Las técnicas específicas incluyen explorar las consecuencias positivas y negativas del consumo y desarrollar estrategias de respuesta y afrontamiento. (National Institute on Drug Abuse, 2010 citado por Manchini, 2018, p. 37). Otro aporte importante es el que brinda Morales et al., (2013) que explica que el entrenamiento de habilidades sociales potencia distintas variables como empatía, prosocialidad, asertividad y las habilidades cognitivas como la autoeficacia y el afrontamiento. Esta idea se relaciona con lo expuesto por Wolin y Wolin (1993) citado en Machicao \& Aillon (2009) que menciona a la capacidad para relacionarse como pilar de la resiliencia y lo define como la habilidad para establecer lazos íntimos y satisfactorios con otras personas.

La psicoeducación es otra variable que fomenta el desarrollo de la resiliencia ya que permite a los 
pacientes la posibilidad de desarrollar y a la vez fortalecer sus capacidades con la finalidad de afrontar las situaciones conflictivas o que le resulten de alguna manera problemática de un modo más adaptativo (Roca, 2015 citado en Lucas, 2019). Así también lo manifiesta Haro, Ramírez et al., (2006) citado en Rubio, Begoña et al., (2105) que explica que el aumento en el conocimiento o la conciencia por medio de la psicoeducación puede favorecer a una mejor comprensión del problema que se traduciría en sensación de control y autoeficacia ante las adversidades.

Otra estrategia que desarrolla resiliencia es el autocontrol que según Carrasco citado por Labrador (2009) citado por Midrigal \& Marin, (2013) se relacionan con la capacidad de cambiar las conductas consideradas inapropiadas, contiene criterios que buscan fomentar estrategias para lograr regular los comportamientos que contribuyen con lograr metas establecidas. EI autocontrol emocional también permite tener el equilibrio necesario para tomar decisiones y conseguir la adecuada activación interna para afrontar las adversidades diarias (Peralbo, 2011, pág. 28). También se destacan a las técnicas de relajación que son otra opción para trabajar y reducir altos niveles de ansiedad, producto de la percepción de amenaza que se le otorgan a los estímulos externos, por lo tanto, podrían brindar estrategias de afrontamiento para disminuirla (Caballo, 1991 citado por Midrigal \& Marin, 2013). A su vez, Labrador et al. (2004) citado por Midrigal \& Marin, (2013) mencionan que una técnica de relajación es "cualquier procedimiento cuyo objetivo sea enseñarle a la persona a controlar su propio nivel de activación sin ayuda de recursos externos" permitiendo su utilización en situaciones estresantes.

Para desarrollar estrategias resilientes es importante la construcción del proyecto de vida, ya sea en la adolescencia o la edad adulta, ya que manejan formatos y se trabaja en puntos específicos en cada etapa de vida. Por lo tanto, en este caso, es necesario planificarlo con anticipación para fortalecer los puntos clave de la personalidad del individuo, tales como: autoconcepto, autoestima, habilidades sociales, 
toma de decisiones $y$ establecimiento de metas. (Aguirre, 2016, p. 11). El deporte es otro factor generador de resiliencia, pues tal y como establece Jürgens (2006) citado por Chacón et al., (2016), la práctica deportiva otorga oportunidades de relacionarse, de resolver conflictos, genera independencia y confianza en sí mismo, además de iniciativa, optimismo y pensamiento crítico (Dramismo, 2007) citado por (Chacón et al., 2016). También se observó la aplicación de distintas actividades donde se fomenta la iniciativa, la introspección y la creatividad, características de la resiliencia propuestas por Wolin y Wolin (1993), citado por Machicao \& Aillon (2009) en quienes ven a la iniciativa al explorar el entorno o nuevas experiencias hasta el emprender nuevos proyectos. También a la creatividad, a través del juego dándole un significado a las experiencias traumáticas, otorgándole un nuevo sentido a la vida y la introspección o insight, como capacidad para reflexionar sobre las acciones y asumir una postura ética frente a ella.
En relación con los datos obtenidos en el test SV-RES, podemos observar que no existe diferencia significativa en el aumento de los niveles de resiliencia entre los primeros tres meses de tratamiento y en los siguientes tres meses de duración del proceso de rehabilitación, donde predomina el $60 \%$ de los pacientes con un nivel medio en resiliencia al igual que los primeros tres meses, determinando que no ha existido una eficacia considerable en el desarrollo de las estrategias resilientes y que al implementar nuevas estrategias, junto con el aumento de la frecuencia con la que se realizan estas intervenciones, se puede aumentar los niveles de resiliencia en los pacientes del centro de rehabilitación.

Para que existan mayores niveles de resiliencia también es importante aumentar las redes de apoyo de los familiares y amigos de los pacientes en el programa de rehabilitación, como menciona Werner \& Johnson (2004) citado por Cabrera (2019) que las personas en consumo de sustancias sienten satisfacción y son más resilientes gracias al apoyo de su madre, hermanos y amigos de la 
infancia. Ese comportamiento resiliente, lleva a los individuos a elevar la autoestima y la autoeficacia a través de las relaciones de apoyo. Se puede destacar que este factor de la resiliencia presenta uno de los porcentajes más bajos junto con la satisfacción en los resultados del test SV-RES y se ve afectado por diferentes variables como las familias disfuncionales de donde provienen algunos pacientes 0 las políticas del programa de impedir comunicación con personas que no participan del programa hasta después de un determinado tiempo. Es recomendable la orientación familiar de los pacientes, mejorar la eficacia en las intervenciones, aumentando el personal multidisciplinario, realizando las actividades ya descritas con más frecuencias y creando nuevas estrategias resilientes para mejorar los niveles de resiliencia.

Los resultados de esta investigación concuerdan con lo propuesto por Meza \& Delgado (2019) donde los resultados del estudio determinan que los niveles de resiliencia en los pacientes con más de tres meses en el proceso de recuperación se encuentran en su gran mayoría en nivel medio y se han fortalecido de acuerdo con el tiempo que llevan en rehabilitación.

\section{Conclusiones}

La resiliencia es un pilar fundamental en la vida de cada ser humano, ya que permite tener la capacidad de afrontar las adversidades que se nos presenta en la vida, evitando el estancamiento y los pensamientos negativos que nos suelen aturdir cuando no sabemos qué hacer ante situaciones difíciles.

El centro de rehabilitación Nueva Esperanza cuenta con una serie de actividades que fomentan la resiliencia en los pacientes, sin embargo, constatando los resultados del test SV-RES no se observa un aumento considerable en los niveles de resiliencia de los pacientes. También se identificaron los resultados de los factores de resiliencia donde existen niveles bajos producto de la falta de las redes de apoyo o afecto de los familiares, por esta razón es importante seguir fomentando la resiliencia mediante intervenciones y técnicas innovadoras y utilizarlas con más frecuencia para mejorar los 
niveles de resiliencia en los pacientes de este centro de rehabilitación

Se puede afirmar que el centro de rehabilitación Nueva Esperanza, fue un facilitador importante para identificar las estrategias resilientes mediante las diversas actividades y formas de intervención como las terapias individuales, grupales y vivenciales para dar a conocer la estructura y forma de trabajo con los pacientes, evidenciando que se debe seguir trabajando en otras áreas, con otras técnicas y formas de intervención para fomentar la resiliencia en sus pacientes, mejorar el tratamiento y prevenir posibles recaídas.

\section{Bibliografía}

Aguirre, R. (2016). Construcción de un proyecto de vida en adolescentes que consumen drogas. Un estudio de casos. (Tesis de pregrado). Universidad del Azuay. Cuenca-Ecuador.

Recuperado de http://dspace.uazuay.edu.ec/ bitstream/datos/5549/1/11878 .pdf

Ariza, J., Rubio, L., \& Russi, L., (2015). Estrategias de afrontamiento para fortalecer la Resiliencia en población drogodependiente. Colombia, Bogotá: Recuperado de https://repository.ucc.edu.co/ bitstream/20.500.12494/1308 0/1/2015_estrategias_afronta miento_resiliencia.pdf

Apolo Morán, J. F., Rivas Maldonado, N. E., \& Cucalón Franco, P. (2017). Más allá de los 12 pasos. Tratamiento de adicciones desde una visión profesional. Diagramación, Ecuador-Guayaquil, Recuperado de https://0201.nccdn.net/1_2/00 0/000/0b5/272/Tatamientoprofesional-paraadicciones.pdf

Cabrera, R. (2019) Análisis de la Resiliencia en personas drogodependientes (Tesis de posgrado). Universidad de la Laguna. Cristóbal de La Laguna, España. Recuperado de

https://riull.ull.es/xmlui/bitstre am/handle/915/17180/Analisi s\%20de\%20la\%20Resiliencia \%20en\%20personas\%20drog odependientes..pdf?sequenc $\mathrm{e}=1$

Cano Valle, F., Nanni Alvarado, R., \& Pantoja Nieves, M. (2016). Derechos de las Personas con Enfermedades Psiquiátricas. México: INEHRM. Recuperado de https://archivos.juridicas.una m.mx/www/bjv/libros/9/4327/1 1.pdf 
Callisaya Argani, Juana Isabel. (2017). Análisis de la resiliencia de un adolescente en situación de riesgo por consumo de alcohol. Fides et Ratio - Revista de Difusión cultural y científica de la Universidad La Salle en Bolivia, 13(13), 41-58. Recuperado de http://www.scielo.org.bo/sciel o.php?script=sci_arttext\&pid= S2071081X2017000100004\&lng=es \&tlng=es.

Campos, D., Odar, E. (2018). Programa desde el enfoque cognitivo conductual en la resiliencia en internos drogodependientes de un centro de rehabilitación. (Tesis de grado). Universidad señor de Sipán. Pimentel, Perú. Recuperado de http://repositorio.uss.edu.pe/b itstream/handle/uss/5476/Ca mpos $\% 20$ Heredia\%20\%26\% 200dar\%20Regalado.pdf?se quence $=1$ \&isAllowed $=y$

Chacón Cuberos, Ramón, \& CastroSanchez, Manuel, \& EspejoGarcés, Tamara, \& Zurita Ortega, Felix (2016). Estudio de la resiliencia en función de la modalidad deportiva: fútbol, balonmano y esquí. RETOS. Nuevas Tendencias en Educación Física, Deporte y Recreación, (29),157-161.

ISSN:

1579-1726.

Recuperado de https://www.redalyc.org/art iculo.oa?id=3457/345743464 032

Cruz, M. (2016). Asertividad y resiliencia en atletas (Tesis de grado) Universidad Rafael Landívar. Quetzaltenango, Guatemala. Recuperado de http://186.151.197.48/tesiseor tiz/2016/05/42/Cruz-Mario.pdf

Farias, L., Guerra, V., Cifuentes, T., \& Rozas, S. (2010). Consumo problemático de drogas y Terapia Ocupacional: Componentes ocupacionales evaluados durante el proceso de tratamiento $y$ rehabilitación. Revista Chilena de Terapia Ocupacional, Volumen (10), 45-56. Recuperado de file:///C:/Users/Usuario/Downl oads/10559-1-23356-1-1020101230.pdf

García-Vesga, M. C. \& Domínguezde la Ossa, E. (2013). Desarrollo teórico de la Resiliencia y su aplicación en situaciones adversas: Una revisión analítica. Revista Latinoamericana de Ciencias Sociales, Niñez y Juventud, 11 (1), pp. 63-77. Recuperado de

https://www.redalyc.org/pdf/7 73/77325885001.pdf

Jiménez, Y., \& Matute, D., (2018). Resiliencia en pacientes adictos internos en centros de atención terapéutica de la 
ciudad de Cuenca. EcuadorCuenca: Recuperado de https://dspace.ucuenca.edu.e c/bitstream/123456789/30215 /1/Trabajo\%20de\%20titulaci \%C3\%B3n.pdf

Lizano Moya, K. A., (2017). Resiliencia y Psicodrama. (Tesis de pregrado). Universidad San Francisco de Quito usfq, Quito, Ecuador. Recuperado de http://repositorio.usfq.edu.ec/ bitstream/23000/6585/1/1311 36.pdf

Lucas, K. (2019). Psicoeducacion y su aporte terapéutico en la reinserción social de un paciente. (Tesis de pregrado). Universidad Técnica de Babahoyo. Babahoyo, Ecuador. Recuperado de http://dspace.utb.edu.ec/bitstr eam/handle/49000/6537/EUTB-FCJSE-PSCLIN000191. pdf? sequence $=1$

Machicao Arauco, Adriana, \& Aillón Soria, Susana. (2009). Fortalecimiento de factores protectores de la resiliencia en el ámbito comunitario en mujeres en situación de violencia doméstica. Ajayu Órgano de Difusión Científica del Departamento de Psicología UCBSP, 7(1), 127. Recuperado de http://www.scielo.org.bo/sciel o.php?script=sci_arttext\&pid= S2077-
$21612009000100001 \& \operatorname{lng}=\mathrm{es}$ \&tlng=es.

Madrigal Monge, J., Marin, K. (2013). Efectos del entrenamiento en técnicas de relajación y de autocontrol sobre la ansiedad de un grupo de hombres con dependencia al alcohol en remisión sostenida. (Tesis de pregrado) Universidad de Costa Rica. Limón, Costa Rica. Recuperado de http://163.178.170.204/bitstre am/handle/123456789/56/JU LIETTE\%2035647.pdf?seque nce $=1$ \&isAllowed $=y$

Manchini, D. (2018). "Perfil resiliente en un grupo de personas que se encuentran transitando la última etapa y las que han finalizado el tratamiento del grupo institucional de alcoholismo." (Tesis de pregrado). Pontificia universidad católica argentina. Recuperado en: https://repositorio.uca.edu.ar/ bitstream/123456789/587/4/p erfil-resiliente-grupopersonas.pdf

Meza, A. K. T., \& Delgado, J. J. S. (2019). Resiliencia en pacientes drogodependientes de la Comunidad Terapéutica "Camino a La Paz". Espirales Revista Multidisciplinaria de investigación, 3(30). Recuperado de file:///C:/Users/PC/Downloads /596-1873-4-PB\%20(11).pdf 
Morales, M., Benítez, M. y Agustín, D. (2013). Habilidades para la vida (cognitivas y sociales) en adolescentes de zona rural. Revista Electrónica de Investigación Educativa, 15(3), 98-113. Recuperado de http://redie.uabc.mx/vol15no3 /contenido-moralesetal.html.

Pimentel, J. Telumbre, J. Ruiz, G. Higuera, J. B. T. (2015). Autoestima y consumo de alcohol en el adolescente de Chilpancingo Guerrero, México. [Tesis doctoral]. Lugar de publicación: Editorial; año. Revista de Psicología y Ciencias Del Comportamiento de La U.A.C.J.S.

Prochaska, J., Redding, C. y Evers, K. (2015). The transtheoretical model and stages of change. In Behavior and health education: theory, research, and practice (pp. 125-148).

Saavedra, E., Villalta, M. (2008). Escala de Resiliencia SV-RES para jóvenes y adultos (CEANIM ed.). Recuperado de https://www.academia.edu/26 343874/Saavedra_-

Villalta SV

RES60_ESCALA_DE_RESIL IENCIA_SV-RES

Saavedra, G., Villalta, P. (2008b). Medición de las características resilientes, un estudio comparativo en personas entre 15 y 65 años. Liberabit, (14), 31-40. Disponible en: http://www.scielo.org.pe/pdf/li ber/v14n14/a05v14n14.pdf

Redondo-Elvira, Tamara, Ibañezdel-Prado, Celia, \& BarbasAbad, Sara.

(2017). Espiritualmente resilientes. Relación entre espiritualidad y resiliencia en cuidados paliativos. Clínica y Salud, 28(3), $\quad 117$ 121. https://dx.doi.org/10.101 6/j.clysa.2017.09.001

Secretaria técnica de Prevención Integral de Drogas. (2017). Plan Nacional de Prevención Integral y Control del Fenómeno Socio Económico de las Drogas. Recuperado de http://www.cicad.oas.org/Fort alecimiento_Institucional/plan esNacionales/Ecuador_Plan_ Nacional_de_Prevencion_Int egral_y_Control_del_Fen\%C 3\%B3meno_Socio_Econ\%C3 \%B3mico_de_las_Drogas_20 17_2021.pdf

Villalba, C. (2004). El concepto de la resiliencia individual y familiar. Aplicaciones en la intervención social. Revista del Colegio Oficial de Psicólogos de Madrid. Recuperado de http://site.ebrary.com/lib/bibsi pansp/docDetail.action 